\title{
Breath Hold Breast Radiotherapy In The Era of Precision Medicine: Appropriate Selection for Left Descending Artery Sparing
}

Edy Ippolito

Campus Bio-Medico University: Universita Campus Bio-Medico di Roma

Carlo Greco ( $\nabla$ c.greco@unicampus.it )

Policlinico Universitario Campus Bio-Medico https://orcid.org/0000-0002-1068-5045

\section{Maristella Marrocco}

Campus Bio-Medico University: Universita Campus Bio-Medico di Roma

\section{Silvia Gentile}

Campus Bio-Medico University: Universita Campus Bio-Medico di Roma

\section{Serena Palizza}

Campus Bio-Medico University: Universita Campus Bio-Medico di Roma

\section{Michele Fiore}

Campus Bio-Medico University: Universita Campus Bio-Medico di Roma

\section{Luca Eolo Trodella}

Campus Bio-Medico University: Universita Campus Bio-Medico di Roma

\section{Rolando Maria D'Angelillo}

University of Rome Tor Vergata: Universita degli Studi di Roma Tor Vergata

\section{Sara Ramella}

Campus Bio-Medico University: Universita Campus Bio-Medico di Roma

\section{Study protocol}

Keywords: DIBH, breast cancer, left descending artery

Posted Date: December 15th, 2020

DOl: https://doi.org/10.21203/rs.3.rs-127934/v1

License: (c) (i) This work is licensed under a Creative Commons Attribution 4.0 International License. Read Full License 


\section{Abstract \\ Background and purpose}

To identify anatomical and/or preplanning characteristics correlated with left descending artery (LAD) dose and therefore provide guidance in the selection of patients with left-breast cancer that could benefit the most from the use of deep inspiration breath hold radiotherapy (DIBH-RT).

\section{Materials and methods}

We retrospectively identified patients with left-sided breast cancer who underwent whole breast radiotherapy in DIBH and extracted data from treatment plans in free-breathing (FB) and DIBH. The following anatomical parameters were obtained from the planning CTs in FB: lung volume, heart volume, breast separation, minimum distance from LAD to tangent open field. Receiving operating characteristics was also performed to define the cut-off point of parameters to use in LAD dosimetry prediction. Areas under the curve (AUCs) were calculated for all variables. Post-test probability has been calculated to evaluate advantage for parameters combination.

\section{Results}

One hundred ninety-seven patients were identified. The strongest predictor at FB CT scan of LAD maximum dose $>10$ Gy and a LAD mean dose $>4$ Gy was the minimum distance of LAD from tangent open fields. Adding consecutively other preplanning anatomic parameters, the positive predictive value (PPV) to identify patients at risk of higher dose to LAD was $>90 \%$.

\section{Conclusions}

The dosimetric benefit of DIBH is valid for all patients and DIBH should be preferred for all left sided patients; however we can identify a subgroup of patients who benefit the least from DIBH. This is the group with favorable anatomy: limited breast separation, well expanded lungs, LAD distant from open tangent fields.

\section{Introduction}

Adjuvant radiotherapy is a standard of care in treatment of breast cancer in patients undergoing both breast-conserving (BCT) or mastectomy, improving loco-regional control and overall survival [1, 2].

Despite the benefits of RT, left-sided breast cancer radiotherapy showed a higher incidence of adverse cardiac health effects, occurring even many years after treatment and even causing premature mortality. Cardiovascular mortality from left-sided RT is significantly higher compared with right-sided RT and is 
more evident after $\geq 15$ years of follow-up (RR: 1.23, 95\% Cl: 1.08-1.41, P<0.001) [3]. The development of these long-term cardiovascular toxicities seems to be caused by microvascular endothelial injury and myocardial remodeling, as well as oxidative stress and inflammation. This damage has shown to be related to incidental cardiac dose linearly associated with increased risk of major coronary events $[4,5]$. Incidental cardiac dose appears to also be related to the risk of early post radiotherapy perfusion defects on functional imaging [6].

Left descending artery (LAD) irradiation has been increasingly recognized as a relevant mechanism of cardiac damage in preclinical [7 ]and clinical studies. In fact, left sided breast cancer patients showed myocardial perfusion changes in the LAD distribution [8] and LAD stenosis measured by angiography was more common in left sided than right sided breast cancer patients undergoing adjuvant radiotherapy [9]. In addition, both LAD maximum and mean dose were associated with coronary artery calcium score > $0[10]$ and women receiving mean doses between 1-5Gy to the mid LAD had an increased risk for a later coronary intervention compared to women receiving mean doses of 0-1Gy [11] .

Nowadays, there are several radiation technique that allow heart sparing: those that reduce treatment volume to tumor bed (partial breast irradiation, $\mathrm{PBI}$ ); those able to decrease the delivery of higher dose to the heart, using intensity modulated radiation therapy (IMRT) or volumetric arc therapy (VMAT); and those that reduce cardiac exposure to radiation by moving the heart away from the target, such us prone position or deep inspiration breath hold (DIBH). Particularly, the latter increases the physical separation between the chest wall and the heart during inspiration and has recently been recommended as the best heart sparing technique available [12]. Literature data reports a reduction of mean heart dose ranging from $38-59 \%$ and a reduction of mean dose to left descending artery (LAD) ranging from $31-71 \%$ with $\mathrm{DIBH}[13]$.

However, even if the benefit from this technique is clearly recognized, not all patients achieve the same degree of benefit. Therefore, as not all patients are compliant in performing $\mathrm{DIBH}$, showing different degrees of cooperation, as the treatment itself prolongs simulation and treatment delivery times increase staff workload, patient commitment and treatment cost, it would be useful to identify predictors of dosimetric benefit, especially in the context of limited resources.

Several studies have mainly evaluated anatomical predictors of mean heart dose (MHD) reduction with $\mathrm{BH}$ technique $[13,14]$. However, mean heart dose does not seem to be representative of dose to the $L A D$ [15].

In fact in the BACCARAT study, among left-sided breast cancer patients with a mean heart dose lower than $3 \mathrm{~Gy}$, more than half of patients could receive dose greater than $40 \mathrm{~Gy}$. Therefore the authors concluded that the dose delivered to the cardiac substructures, such as the LAD, should also be assessed [15].

Based on the above considerations, the aim of this study was to identify in a large dataset of patients anatomical and/or treatment preplanning characteristics correlated with LAD dose in order to guide 
selection of patients with left-breast cancer that could benefit the most from the use of DIBH-RT.

\section{Methods And Material Patient selection}

We retrospectively identified patients with left-sided breast cancer treated with whole breast adjuvant radiotherapy (WBRT) in DIBH at our institute from 2014 to 2018. As we routinely have FB contingency plans for all patients treated with $\mathrm{DIBH}$, we performed a quantitative retrospective analysis of dosimetric data from treatment plans in free-breathing (FB) and DIBH for each patient. Patients gave informed consent for the use of clinical ad image data for research purpose.

\section{Simulation, contouring and treatment planning}

All patients were positioned supine on a breast board with both arms raised above the head. Each patient received a training session to establish the individual deep inspiration level. Patients who were not able to maintain a stable deep inspiration breath hold for at least 20 seconds were deemed not eligible for DIBH technique. First patients underwent a FB CT scan immediately followed by a DIBH CT scan in the same position. The scan was extended from the jugular notch to $5 \mathrm{~cm}$ below the lower edge of the breast with a scan interval of $5 \mathrm{~mm}$. The target volume, the heart, left anterior descending artery (LAD), contralateral breast and ipsilateral lung were manually contoured on each CT slice by a radiation oncologist with at least 5 years' experience, following the RTOG guidelines [16] and heart atlas published by Feng et al [17]. Target volumes and OARs were contoured using Eclipse Treatment Planning System (TPS). The total prescribed dose was $50 \mathrm{~Gy}$ in 25 fractions. Treatment plans consisted of a simple wedged tangential plan (with gantry angles optimized to match divergence of the posterior edges of the beam) to avoid contralateral irradiation and to minimize the ipsilateral lung and heart area. Moreover, in some cases a field in field technique or irregular surface compensator technique was also applied.

\section{Anatomical and treatment planning data}

The following anatomical parameters were obtained from the planning CTs in FB: lung volume (cc), heart volume (cc), breast separation (cm), minimum distance from LAD to tangent open field. The last one was the shortest distance measured between LAD and tangent open fields on both axial and sagittal scan planes. Heart dosimetric data collected from both plans (FB and DIBH) for each patient were mean LAD dose and maximum LAD dose. Furthermore, the differences of these values $(\Delta, \%)$ between FB and DIBH plans were also calculated.

\section{Statistical analysis}

Continuous variables were reported as mean and standard deviation. Comparison between the dosimetric data of the two techniques (FB and VDIBH) was performed by means of the non-parametric MannWhitney's test ( $p$ value $<0.05$ were considered as significant). We identified a LAD maximum dose $\leq$ $10 \mathrm{~Gy}$ and a LAD mean dose $\leq 4 \mathrm{~Gy}$ as clinical goal. In fact, most patients (except for the upper quartile) 
treated with $\mathrm{BH}$ technique received doses lower than this. We evaluated which anatomical variables in FB scan were predictors of LAD dose higher than $10 \mathrm{~Gy}$ (maximum dose) and $4 \mathrm{~Gy}$ (mean dose). Receiving operating characteristics (ROC analysis) was also performed to define the cut-off point of anatomical parameters to use in LAD dosimetry prediction. Areas under the curve (AUCs) were calculated for all variables. Post-test probability has been calculated to evaluate advantage for anatomical parameters combination [18]. The Med-Calc 11.6.1.0 statistical package (MedCalc Software, Mariakerke, Belgium) was used for all statistical analysis.

\section{Results}

One hundred ninety-seven patients were identified and data from 394 treatment plans was extracted and analyzed.

\section{Dosimetric comparison}

Median, mean and interquartile range values of mean and maximum LAD dose in both plans are summarized in table 1. All values were significantly reduced in BH plans (figure 1). In particular, maximum and mean dose to $L A D$ were reduced by $31.7 \%$ (mean value, $S D=22$ ) and $28.1 \%$ (mean value, $\mathrm{SD}=17)$ in $\mathrm{BH}$ plans compared to FB plans.

\section{ROC curve analysis}

By ROC curve analysis, several anatomic variables measured in the FB scan (minimum distance from $L A D$ to tangent open fields, heart volume, lung volume, breast separation) have been independently tested to predict for a LAD maximum dose $>10 \mathrm{~Gy}$ and for a LAD mean dose $>4 \mathrm{~Gy}$. For LAD maximum dose > $10 \mathrm{~Gy}$, cut off value of minimum distance from LAD to tangent fields resulted $\leq-0.1 \mathrm{~cm}$ whereas for LAD mean dose $>4 \mathrm{~Gy}$ the cut-off was increased $(<0.49 \mathrm{~cm})$. Otherwise, similar cut off values of heart and lung volume (> $650 \mathrm{cc}, \leq 1100 \mathrm{cc}$ respectively) could predict both mean ( $>4 \mathrm{~Gy}$ ) and maximum LAD dose (> $10 \mathrm{~Gy}$ ). Table 2 summarizes AUCs for each variable cut point (only AUCs of variables with a $p$ value $<0.1$ are shown). ROC comparison curves of variables at FB scans predicting a LAD maximum dose $>10$ Gy and a LAD mean dose $>4$ are shown in figure 2 .

\section{Post-test probability analysis}

The minimum distance of LAD from tangent open fields was the strongest predictor of receiving a LAD maximum dose (Dmax) $>10$ Gy and a LAD mean dose (Dmean) $>4$ Gy was. For detecting a LAD maximum dose $>10$ Gy the AUC was $0.801(95 \% \mathrm{Cl}: 0.649-0.908)$ with an odd ratio approximately 4 times higher when the minimum distance is lower than $-0.1 \mathrm{~cm}(\mathrm{OR}=4.363 ; 95 \% \mathrm{IC}: 1.80-10.53, \mathrm{p}=0.0010)$. The positive predictive value to discriminate patients with a LAD maximum dose $>10 \mathrm{~Gy}$ was $73 \%$, if the minimum distance of $L A D$ from tangent field was $\leq-0.1 \mathrm{~cm}$. Adding consecutively other parameters such as heart volume greater than $655.5 \mathrm{cc}$ and lung volume lower than or equal to $1087.7 \mathrm{cc}$, the positive predictive value (PPV) was increased from $73 \%$ to $91 \%$ (see Table 3). For detecting a LAD mean dose $>4$ 
Gy the AUC was $0.715(95 \% \mathrm{Cl}: 0.553-0.845)$ with an odd ratio of approximately 5 times higher when the minimum distance is lower than or equal to $0.5 \mathrm{~cm}(\mathrm{OR}=: 4.9687 ; 95 \% \mathrm{IC}: 2.0662-11.9486, \mathrm{p}=0.0003)$. In fact, the minimum distance of LAD from tangent open fields $<0.49 \mathrm{~cm}$ showed a positive predictive value of $79 \%$. Adding consecutively other parameters such as heart volume greater than $>652.6 \mathrm{cc}$, a lung volume $<1190.6$ and a breast separation $>16.1 \mathrm{~cm}$, the positive predictive value was increased from $79 \%$ to $98 \%$ (see Table 3 ).

\section{Discussion}

The aim of this study was to identify anatomical and/or pre planning characteristics correlated with LAD dose. Recent guidelines recommend the following dose constraints for LAD: Dmean < 10 Gy; V30 Gy (volume of LAD receiving $\geq 30 \mathrm{~Gy}$ ) $<2 \%$; V40 Gy (volume of LAD receiving $\geq 40 \mathrm{~Gy}$ ) $<1 \%$ [19] However, we chose a cut point of $10 \mathrm{~Gy}$ and $4 \mathrm{~Gy}$ for LAD maximum and mean dose respectively, as $75 \%$ of patients treated with $\mathrm{BH}$ technique in our cohort received lower doses. We, therefore, believed that these cut points could be reasonable for our study purpose.

To the best of our knowledge, this is the largest series investigating the combination of anatomical factors in predicting LAD doses. Register $S$ et al found among 64 patients that only changes in heart volume in field predicted for cardiac sparing in terms of heart mean dose, heart V30Gy, LAD maximum dose and LAD V40 Gy, not finding any other anatomic surrogates for heart dosimetric benefit [14]. Similarly Rochet et al in a small subset of patients found that free breathing cardiac contact distance was a good predictor for cardiac dose [20.] More recently, Cao $\mathrm{N}$ et al provided a very useful prediction model for mean heart dose reduction from a set of 67 consecutive breast cancer patients. Particularly, in this model FB heart- to chest distance and cardiac-contact distance measured in both the axial and sagittal planes were used as anatomic predictors of cardiac sparing [13]. In our study, the minimum distance of LAD from tangent fields was the best anatomical variable measured in FB CT scan able to discriminate patients at higher risk to receive a maximum LAD dose $>10 \mathrm{~Gy}$ and a mean LAD dose of 4 Gy with a PPV of $73 \%$ and $79 \%$ respectively. This simple and easily obtainable parameter ultimately represents the LAD portion more exposed to radiation based on treatment field geometry. Interestingly, not only negative values (LAD within the tangent fields), but also positive values (LAD close to tangent fields) were predictors of higher dose to LAD. Even if several anatomic parameters were meaningfully related with LAD doses (Table 2), only minimum distance of LAD at ROC comparison curves analysis (Fig. 2) retained significance. However, combining the different parameters, the PPV increased from $73 \%$ to $91 \%$ for detecting a LAD maximum dose $>10$ Gy and from $79-98 \%$ for detecting a LAD mean dose $>4$ Gy. Therefore a combination of anatomic factors plays a role in increasing LAD exposure and should be carefully considered.

This study has several limitations. First, this patient population included only patients receiving radiotherapy to the intact breast (WBRT) with tangent 3-D conformal field. Thus, the results cannot be extended to patients receiving post-mastectomy radiotherapy and to patients treated with intensity modulated radiotherapy (IMRT) or volumetric arc therapy (VMAT). Second, all measurements were 
collected retrospectively and, considering inter and intra-observer variability in the contouring of the LAD, this could determine unseen biases. For this reason, all LAD contours were reviewed independently by 2 physicians.

On the other hand, the strengths of our study rely on the large amount of data available for the analysis and on the combined effect on dose prediction of different anatomical and preplanning characteristics. This is a novel and interesting finding.

In conclusion, the DIBH plans achieved lower doses to the LAD in all patients; this can be particularly important taking into account that $\mathrm{BH}$ technique suffers less from organ motion than $\mathrm{FB}$ and, therefore, the administered dose to heart and its substructures is more likely to be similar to those of the treatment plan.

DIBH should be the first option when treating left-sided breast cancer patients; however we can identify a subgroup of patients who benefit the least from DIBH. This is the group with favorable anatomy: limited breast separation, well expanded lungs, LAD distant from open tangent fields. This should be taken into consideration for patients selection in a setting of limited resources as $\mathrm{BH}$ technique requires more efforts for the patient and the therapist than FB.

\section{Abbreviations}

left descending artery (LAD)

deep inspiration breath hold radiotherapy (DIBH-RT)

free-breathing (FB)

Areas under the curve (AUCs)

positive predictive value (PPV)

breast-conserving (BCT)

partial breast irradiation (PBI)

intensity modulated radiation therapy (IMRT) or volumetric arc therapy (VMAT)

Treatment Planning System (TPS)

mean dose (Dmean)

Whole breast irradiation (WBRT)

\section{Declarations}


Ethics approval and consent to participate

Ethical approval This retrospective analysis was conducted complying institutional review board requirements. Patients gave informed consent for the use of clinical ad image data for research purpose

\section{Consent for publication}

"Not applicable"

\section{Availability of data and materials}

The datasets used and/or analysed during the current study are available from the corresponding author on reasonable request

\section{Competing interests}

"The authors declare that they have no competing interests"

\section{Funding}

"Not applicable"

\section{Authors' contributions}

\section{- El, SR, CG contributed substantially to the conception of the article;}

- El and MM analyzed and interpreted the patient data regarding the dosimetric parametres.

- El, SG, MM, SS, SP, carried out statistical analysis

- CG, LET, El. SR, SG, SR, SP contributed substantially to collection of clinical informations and data;

- El, CG, SR, LET, SR contributed substantially to review of current literature; contributed substantially to writing of the manuscript and data interpretation;

- El, SR, CG contributed substantially to drafting and revisiting.

All authors have approved the manuscript and agree with its submission to "Radiation Oncology".

\section{- Ethical approval}

This retrospective analysis was conducted complying institutional review board requirements.

Patients gave informed consent for the use of clinical ad image data for research purpose.

\section{References}


1. Early Breast Cancer Trialists' Collaborative Group (EBCTCG), Darby S, McGale P, Correa C, Taylor C, Arriagada R, Clarke M, Cutter D, Davies C, Ewertz M, Godwin J, Gray R, Pierce L, Whelan T, Wang Y, Peto R. Effect of radiotherapy after breast-conserving surgery on 10-year recurrence and 15year breast cancer death: meta-analysis of individual patient data for 10,801 women in 17 randomised trials. Lancet. 2011 Nov 12;378(9804):1707-16. doi: 10.1016/S0140-6736(11)61629-2

2. EBCTCG (Early Breast Cancer Trialists' Collaborative Group), McGale P, Taylor C, Correa C, Cutter D, Duane F, Ewertz M, Gray R, Mannu G, Peto R, Whelan T, Wang Y, Wang Z, Darby S. Effect of radiotherapy after mastectomy and axillary surgery on 10-year recurrence and 20-year breast cancer mortality: meta-analysis of individual patient data for 8135 women in 22 randomised trials. Lancet. 2014 Jun 21;383(9935):2127-35. doi: 10.1016/S0140-6736(14)60488-8

3. Sardar P, Kundu A, Chatterjee S, Nohria A, Nairooz R, Bangalore S, Mukherjee D, Aronow WS, Lavie CJ. Long-term cardiovascular mortality after radiotherapy for breast cancer: A systematic review and meta-analysis. Clin Cardiol. 2017 Feb;40(2):73-81. doi: 10.1002/clc.22631.

4. Giordano SH, Kuo YF, Freeman JL, Buchholz TA, Hortobagyi GN, Goodwin JS. Risk of cardiac death after adjuvant radiotherapy for breast cancer. J Natl Cancer Inst. 2005 Mar 16;97(6):419-24. doi: $10.1093 /$ jnci/dji067

5. Darby SC, Ewertz M, McGale P, Bennet AM, Blom-Goldman U, Brønnum D, Correa C, Cutter D, Gagliardi G, Gigante B, Jensen MB, Nisbet A, Peto R, Rahimi K, Taylor C, Hall P. Risk of ischemic heart disease in women after radiotherapy for breast cancer. N Engl J Med. 2013 Mar 14;368(11):987-98. doi: 10.1056/NEJMoa1209825.

6. Kaidar-Person O, Zagar TM, Oldan JD, Matney J, Jones EL, Das S, Jensen BC, Zellars RC, Wong TZ, Marks LB. Early cardiac perfusion defects after left-sided radiation therapy for breast cancer: is there a volume response? Breast Cancer Res Treat. 2017 Jul;164(2):253-262. doi: 10.1007/s10549-0174248-y.

7. Baker JE, Fish BL, Su J, Haworth ST, Strande JL, Komorowski RA, Migrino RQ, Doppalapudi A, Harmann L, Allen Li X, Hopewell JW, Moulder JE. 10 Gy total body irradiation increases risk of coronary sclerosis, degeneration of heart structure and function in a rat model. Int J Radiat Biol. 2009 Dec;85(12):1089-100. doi: 10.3109/09553000903264473

8. Lind PA, Pagnanelli R, Marks LB, Borges-Neto S, Hu C, Zhou SM, Light K, Hardenbergh PH. Myocardial perfusion changes in patients irradiated for left-sided breast cancer and correlation with coronary artery distribution. Int J Radiat Oncol Biol Phys. 2003 Mar 15;55(4):914-20. doi: 10.1016/s0360-3016(02)04156-1

9. Nilsson G, Holmberg L, Garmo H, Duvernoy O, Sjögren I, Lagerqvist B, Blomqvist C. Distribution of coronary artery stenosis after radiation for breast cancer. J Clin Oncol. 2012 Feb 1;30(4):380-6. doi: 10.1200/JC0.2011.34.5900. Epub 2011 Dec 27.

10. Milgrom SA, Varghese B, Gladish GW, Choi AD, Dong W, Patel ZS, Chung CC, Rao A, Pinnix CC, Gunther JR, Dabaja BS, Lin SH, Hoffman KE, Huff JL, Slagowski J, Abe JI, Iliescu CA, Banchs J, Yusuf SW, Lopez-Mattei JC. Coronary Artery Dose-Volume Parameters Predict Risk of Calcification 
After Radiation Therapy. J Cardiovasc Imaging. 2019 Oct;27(4):268-279. doi: 10.4250/jcvi.2019.27.e38.

11. Wennstig AK, Garmo H, Isacsson U, Gagliardi G, Rintelä N, Lagerqvist B, Holmberg L, Blomqvist C, Sund $\mathrm{M}$, Nilsson $\mathrm{G}$. The relationship between radiation doses to coronary arteries and location of coronary stenosis requiring intervention in breast cancer survivors. Radiat Oncol. 2019 Mar 7;14(1):40. doi: 10.1186/s13014-019-1242-z.

12. Duma MN, Baumann R, Budach W, Dunst J, Feyer P, Fietkau R, Haase W, Harms W, Hehr T, Krug D, Piroth MD, Sedlmayer F, Souchon R, Sauer R; Breast Cancer Expert Panel of the German Society of Radiation Oncology (DEGRO). Heart-sparing radiotherapy techniques in breast cancer patients: a recommendation of the breast cancer expert panel of the German society of radiation oncology (DEGRO). Strahlenther Onkol. 2019 Oct;195(10):861-871. doi: 10.1007/s00066-019-01495-w

13. Cao N, Kalet AM, Young LA, Fang LC, Kim JN, Mayr NA, Meyer J. Predictors of cardiac and lung dose sparing in DIBH for left breast treatment. Phys Med. 2019 Nov;67:27-33. doi: 10.1016/j.ejmp.2019.09.240. Epub 2019 Oct 17.

14. Register S, Takita C, Reis I, Zhao W, Amestoy W, Wright J. Deep inspiration breath-hold technique for left-sided breast cancer: An analysis of predictors for organ-at-risk sparing. Med Dosim. 2015 Spring;40(1):89-95. doi: 10.1016/j.meddos.2014.10.005

15. Jacob S, Camilleri J, Derreumaux S, Walker V, Lairez O, Lapeyre M, Bruguière E, Pathak A, Bernier MO, Laurier D, Ferrieres J, Gallocher O, Latorzeff I, Pinel B, Franck D, Chevelle C, Jimenez G, Broggio D. Is mean heart dose a relevant surrogate parameter of left ventricle and coronary arteries exposure during breast cancer radiotherapy: a dosimetric evaluation based on individually-determined radiation dose (BACCARAT study). Radiat Oncol. 2019 Feb 7;14(1):29. doi: 10.1186/s13014-0191234-z.

16. Breast Cancer Atlas for Radiation Therapy Planning: Consensus Definitions. RTOG Radiation Therapy Oncology Group Web site. http://www.rtog.org /CoreLab/ContouringAtlases/BreastCancerAtlas.aspx

17. Feng M, Moran JM, Koelling T, Chughtai A, Chan JL, Freedman L, et al. Development and validation of a heart atlas to study cardiac exposure to radiation following treatment for breast cancer, IJROBP 2011; 79(1): 10-18. doi:10.1016/j.ijrobp.2009.10.058

18. Albert. On the use and computation of likelihood ratios in clinical chemistry. Clin. Chem 28 (5) (1982) 1113-1119.

19. Piroth MD, Baumann R, Budach W, Dunst J, Feyer P, Fietkau R, Haase W, Harms W, Hehr T, Krug D, Röser A, Sedlmayer F, Souchon R, Wenz F, Sauer R.. Heart toxicity from breast cancer radiotherapy: current findings, assessment and Strahlenther Onkol 2019; 195 (1): 1-12.doi: 10.1007/s00066-0181378-z

20. Rochet N, Drake JI, Harrington K, Wolfgang JA, Napolitano B, Sadek BT, Shenouda MN, Keruakous AR, Niemierko A, Taghian AG.. Deep inspiration breath-hold technique in left-sided breast cancer 
radiation therapy: evaluating cardiac contact distance as a predictor of cardiac exposure for patients selection. Pract Radiat Oncol. 2015 (3): e127-e134. Doi: 10.1016/j.prro.2014.08.0003).

\section{Tables}

Table 1.

LAD dose metrics: free-breathing $(\mathrm{FB})$ and breath hold $(\mathrm{BH})$ plans, dose reduction between $\mathrm{FB}$ and $\mathrm{BH}$ plans .

\begin{tabular}{|lll|}
\hline \multicolumn{1}{|l|}{ FB plans } & BH plans \\
\hline LAD mean dose (Gy) & & \\
\hline Mean & 4.8 & 3.5 \\
\hline Median & 4.5 & 3.2 \\
\hline Percentiles & & \\
\hline 25 & 3.6 & 2.6 \\
\hline 50 & 4.5 & 3.2 \\
\hline 75 & 5.5 & 4.1 \\
\hline LAD maximum dose (Gy) & & \\
\hline Mean & 12.8 & 8.2 \\
\hline Median & 11.1 & 7.5 \\
\hline Percentiles & & \\
\hline 25 & 8.5 & 5.7 \\
\hline 50 & 11.1 & 7.5 \\
\hline 75 & 16.9 & 9.7 \\
\hline
\end{tabular}

$\angle A D$ : left descending artery

Table 2. ROC analysis for predictors of LAD Dmax >10Gy and LAD Dmean > 4 Gy 


\begin{tabular}{|llll|}
\hline LAD Dmax $>10 G y$ & AUC & $95 \%$ IC & P value \\
\hline Minimum distance from LAD to tangent fields $\leq-0.11 \mathrm{~cm}$ & 0.714 & $0.614-0.801$ & $<0.0001$ \\
\hline Lung Volume $\leq 1087.7$ & 0.626 & $0.553-0.695$ & 0.002 \\
\hline Heart volume $>655.51 \mathrm{cc}$ & 0.660 & $0.497-0.799$ & 0.07 \\
\hline LAD Dmean $>4$ Gy & AUC & $95 \% \mathrm{IC}$ & P value \\
\hline Minimum distance from LAD to tangent fields $<0.49 \mathrm{~cm}$ & 0.687 & $0.588-0.744$ & 0.001 \\
\hline Breast separation $>16.1 \mathrm{~cm}$ & 0.649 & $0.550-0.740$ & 0.008 \\
\hline Heart volume $>652.6 \mathrm{cC}$ & 0.672 & $0.508-0.810$ & 0.051 \\
\hline Lung volume $\leq 1190.6$ & 0.624 & $0.519-0.722$ & 0.050 \\
\hline
\end{tabular}

Table 3. FB scans predictors of LAD Dmax $>10$ and LAD Dmean $>4$

FB scans predictors of LAD Dmax $>10$

Minimum distance from LAD to tangent fields $\leq-0.1 \mathrm{~cm}$

Minimum distance from $L A D$ to tangent fields $\leq-0.1+$ Heart volume $>655.5 \mathrm{cc}$

Minimum distance from LAD to tangent fields $<-0.1+$ Heart Volume $>655.5 \mathrm{cc}+$ Lung

Volume $\leq 1087.7$

FB scans predictors of LAD Dmean $>4$

Minimum distance from $L A D$ to tangent fields $<0.5 \mathrm{~cm}+$ Breast separation $>16.1 \mathrm{~cm}$

Minimum distance from $L A D$ to tangent fields $<0.5 \mathrm{~cm}+$ Breast separation $>16.1 \mathrm{~cm}+$ Heart volume $>652.6 \mathrm{cc}$ volume $>652.6 \mathrm{cc}+$ Lung volume $\leq 1190.6$

\section{Figures}



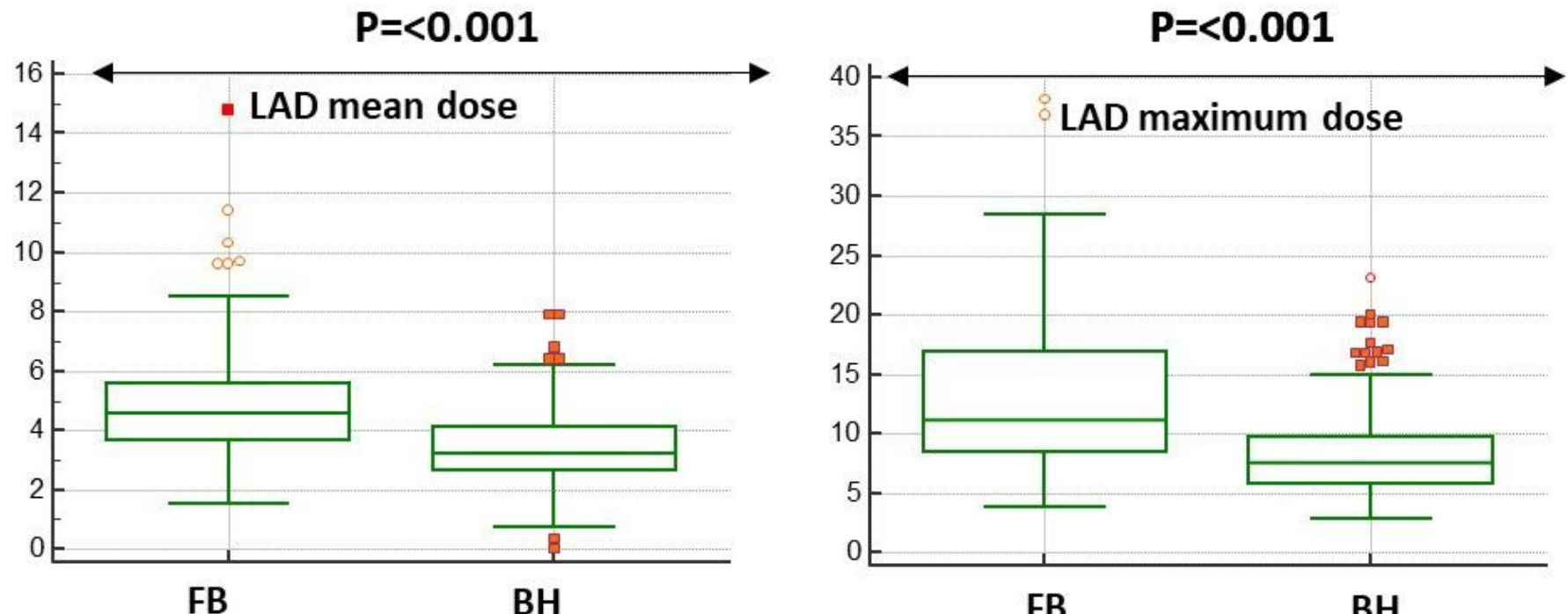

Figure 1

Heart dosimetry comparison between free-breathing and breath hold plans
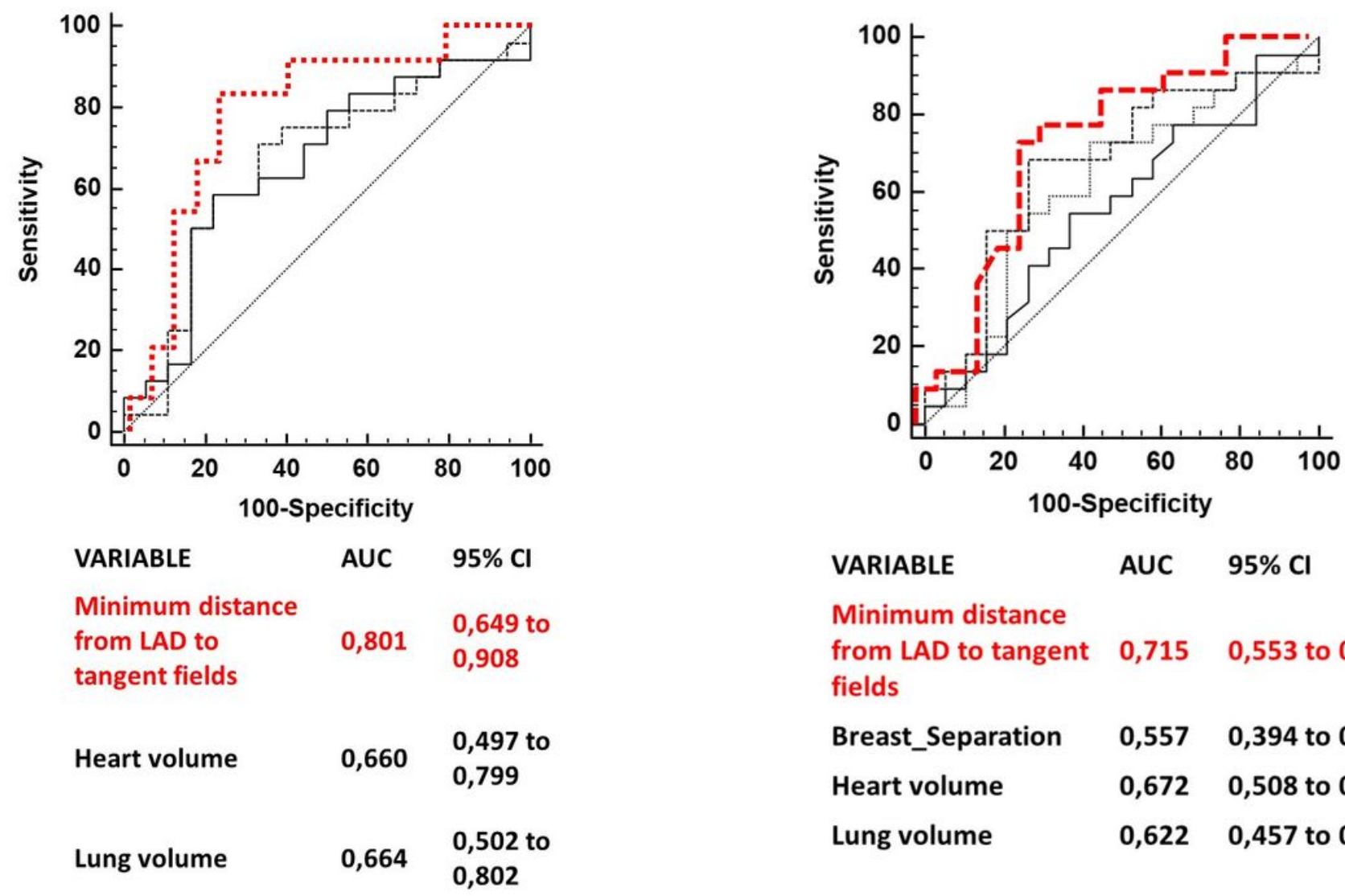

$\begin{array}{lll}\text { VARIABLE } & \text { AUC } & \mathbf{9 5 \%} \mathrm{Cl} \\ \begin{array}{l}\text { Minimum distance } \\ \text { from LAD to tangent } \\ \text { fields }\end{array} & 0,715 & 0,553 \text { to } 0,845 \\ \text { Breast_Separation } & 0,557 & 0,394 \text { to } 0,712 \\ \text { Heart volume } & 0,672 & 0,508 \text { to } 0,810 \\ \text { Lung volume } & 0,622 & 0,457 \text { to } 0,768\end{array}$

Figure 2 
ROC curve comparison of predictors of LAD maximum dose $>10$ Gy (left side) and of LAD mean dose $>4$ Gy (right side) 